X-Ray Absorption Spectroscopy of Uranium Dioxide

J. G. Tobin

December 15, 2010 
This document was prepared as an account of work sponsored by an agency of the United States government. Neither the United States government nor Lawrence Livermore National Security, LLC, nor any of their employees makes any warranty, expressed or implied, or assumes any legal liability or responsibility for the accuracy, completeness, or usefulness of any information, apparatus, product, or process disclosed, or represents that its use would not infringe privately owned rights. Reference herein to any specific commercial product, process, or service by trade name, trademark, manufacturer, or otherwise does not necessarily constitute or imply its endorsement, recommendation, or favoring by the United States government or Lawrence Livermore National Security, LLC. The views and opinions of authors expressed herein do not necessarily state or reflect those of the United States government or Lawrence Livermore National Security, LLC, and shall not be used for advertising or product endorsement purposes.

This work performed under the auspices of the U.S. Department of Energy by Lawrence Livermore National Laboratory under Contract DE-AC52-07NA27344. 


\section{X-ray Absorption Spectroscopy of Uranium Dioxide}

Based upon LLNL-JRNL-454674

\section{Motivation of Analysis}

After the CMMD Seminar by Sung Woo Yu on the subject of the x-ray spectroscopy of UO2, there arose some questions concerning the XAS of UO2. These questions can be distilled down to three issues.

1. The validity of the data.

2. The monchromator energy calibration.

3. The validity of XAS component of the figure shown below.

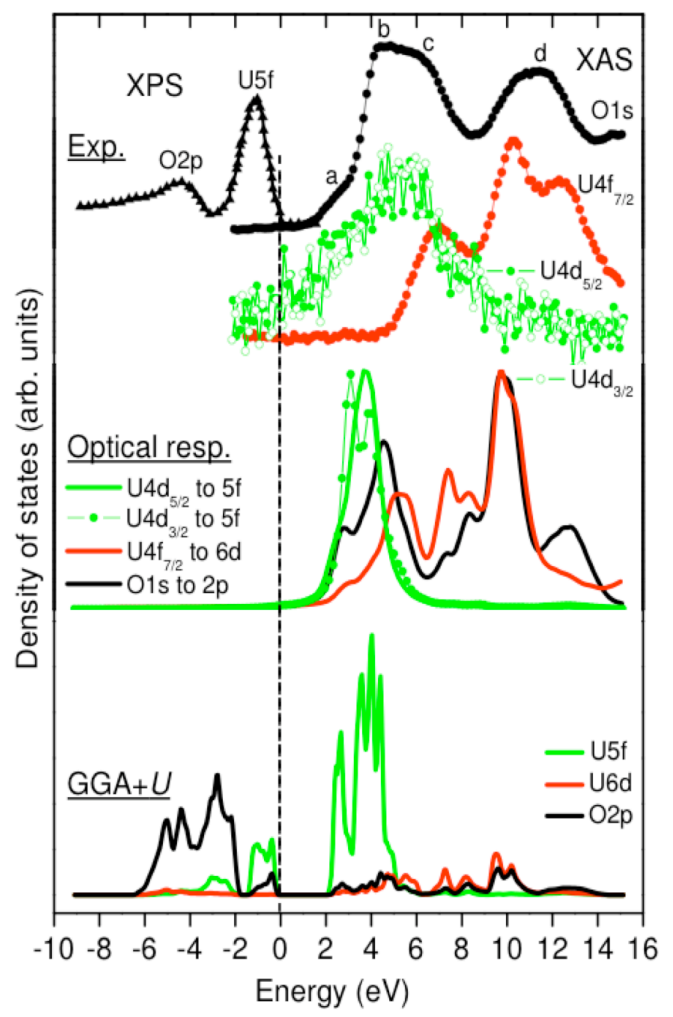

\section{Results of Analysis}

The following will be shown.

1. The data is valid.

2. It is possible to calibrate the monchromator.

3. The XAS component of the above picture is correct.

The remainder of this document is in three sections, corresponding to the three issues. This begins on the next page. 


\section{X-ray Absorption Spectroscopy of Uranium Dioxide}

Based upon LLNL-JRNL-454674

\section{Section 1. Validity of Data}

There were six data ranges probed. They correspond to the $\mathrm{U} 4 \mathrm{~d}(\mathrm{hv}=720-800$ $\mathrm{eV}), 01 \mathrm{~s}(\mathrm{hv}=530 \mathrm{eV}-580 \mathrm{eV})$, the $\mathrm{U} 4 \mathrm{f}(\mathrm{hv}=380 \mathrm{eV}-410 \mathrm{eV})$, the $\mathrm{U} 5 \mathrm{p} 1 / 2(\mathrm{hv}=$ $256-280)$, the U5p3/2 (hv = $174-186 \mathrm{eV})$, the $\mathrm{U} 5 \mathrm{~d}(\mathrm{hv}=90-140 \mathrm{eV})$. The U4d was collected using the high-energy grating and the others were all collected using the middle-energy grating. These are shown below, reordered for ease of comparison.

\section{High Energy Grating U4d Region}

\section{TEY = Total electron Yield TFY = Total Fluorescence Yield}

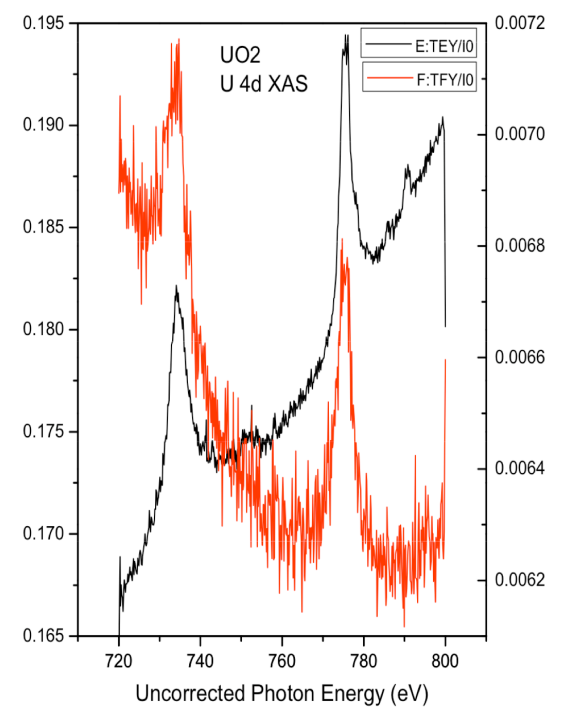

There really isn't any doubt about the validity of the data above. This looks pretty much like the U4d's should. Of course, there is always the issue of backgrounds. Given the constraints placed upon the experimentalists by the ALS, that only a very small sample ( $1 \mathrm{~mm} \times 1 \mathrm{~mm}$ surface area) could be used, background variations and low signal rates are not unexpected. The $\mathrm{x}$-ray beam was larger than the sample and any misalignment could cause contributions from other materials such as the sample holder and sample supports. Thus, what you see above is reasonable.

Kalkowski and Kaindl et al, PRB 35, 2667 (1987).

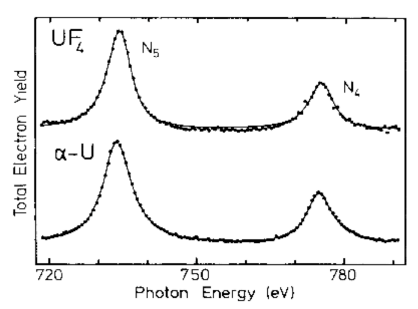

FIG. 7. Total-electron-yield spectra of $\alpha-\mathrm{U}$ metal and $\mathrm{UF}_{4}$ at the $N_{4,5}$ thresholds. The solid lines represent

Kalkowski and Kaindl 's paper is considered the gold standard for XAS of U. As you can see, our U4d's for UO2 fall in the same energy range as theirs for U metal and UF4.

Moore et al, PRB 73, 033109 (2006)

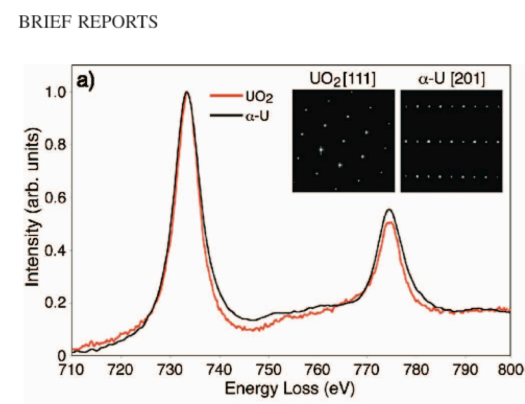

For the sake of completeness, a comparison with UO2 High Energy EELS is included here. Again, our U 4d XAS is consistent with the EELS result. 
Middle Energy Grating

01s Region-01s First Order

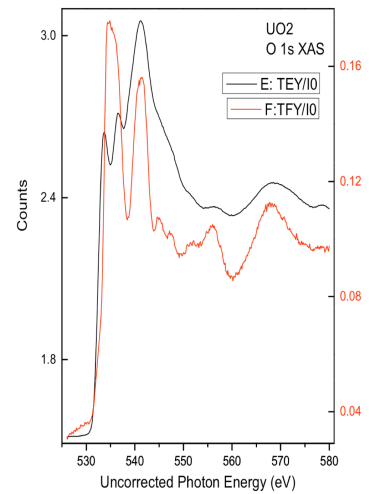

U5p1/2 Region-01s Second Order

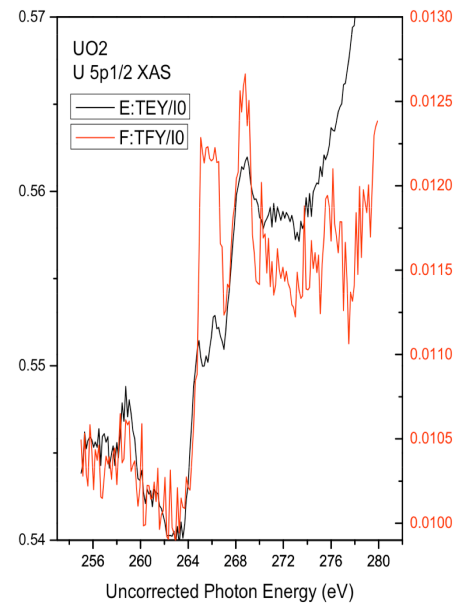

U5p3/2 Region-01s Third Order

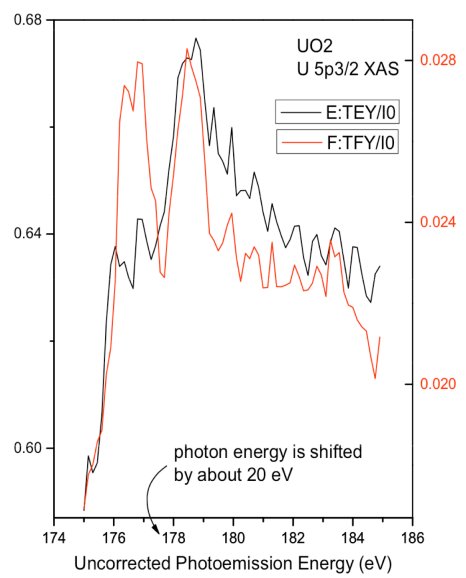

01s, Jollet et al, JPCM 9, 9393 (1997)

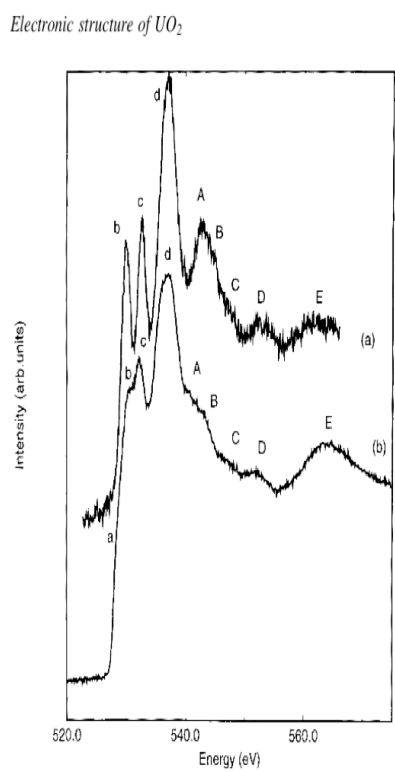

Figure 1. XAS oxygen $\mathrm{K}$ edge of $\mathrm{CeO}_{2}$ (a) and $\mathrm{UO}_{2}$ (b)

\begin{tabular}{llllllllll}
\multicolumn{10}{c}{ Tahle 1. Energies of the structures of the $\mathrm{O}$ K XAS spectrum for $\mathrm{CeO}_{2}$ and $\mathrm{UO}_{2}$. } \\
\begin{tabular}{llllllllll}
\hline & a & b & c & d & A & B & C & D & E \\
\hline $\mathrm{CeO}_{2}(\mathrm{eV})$ & - & 5309 & 533.5 & 537.9 & 543.6 & 545.7 & 548.7 & 553.9 & 563.4 \\
$\mathrm{UO}_{2}(\mathrm{eV})$ & 528.55 & 530.65 & 532.2 & 537.0 & 540.9 & 543.1 & 546.85 & 551.85 & 564.05 \\
\hline
\end{tabular}
\end{tabular}

The bad news: the U5p's are overwhelmed by the $01 \mathrm{~s}$ in $2^{\text {nd }}$ and $3^{\text {rd }}$ order. The good news: this will allow us to calibrate the middle grating perfectly. The other good news, all of our TFY's, $1^{\text {st }}, 2^{\text {nd }}$ and $3^{\text {rd }}$ order, are consistent with each other and all of our TEY's, $1^{\text {st }}, 2^{\text {nd }}$ and $3^{\text {rd }}$ order, are consistent with each other. The TFY's are a better measure of the bulk electronic structure, with TEY's more sensitive to surface modification. However, our TEY's are consistent with the TEY of Jollet et al. The work by Magnuson et al, [Magnuson, Butorin, Werme, Nordgren, Ivanov, Guo, and Shuh, Applied Surface Science 252, 5615 (2006)] for U02 etc is more problematic, possibly representing surface effects in their TEY's. Our TFY's should be a reliable measure of the bulk electronic structure. 


\section{Middle Energy Grating U 4 f Region}

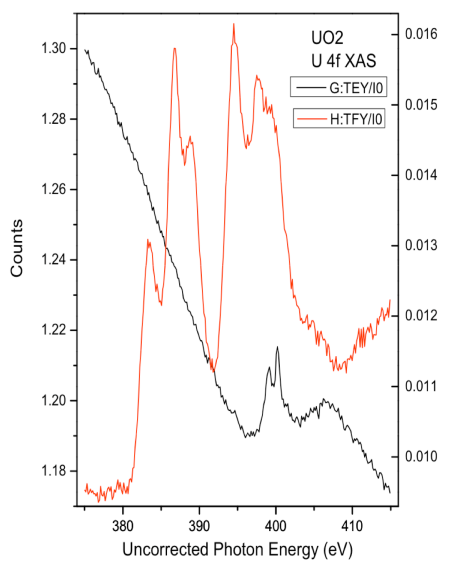

Uranium 4f EELS, Moser, Delley, Schneider and Baer, PRB 29, 2947 (1984)

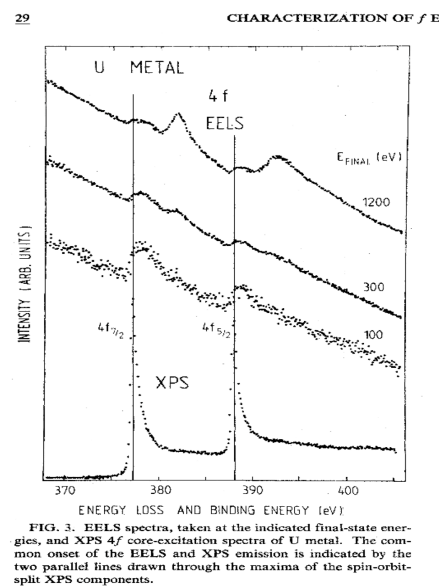

Thorium 4f EELS, Moser, Delley, Schneider and Baer, PRB 29, 2947 (1984)

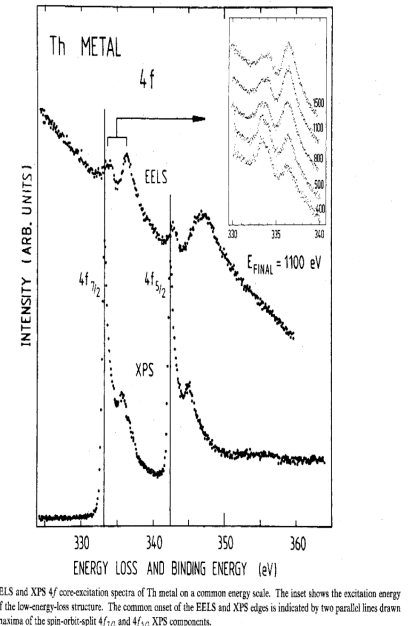

Moore et al, PRB 69, 193104 (2004)

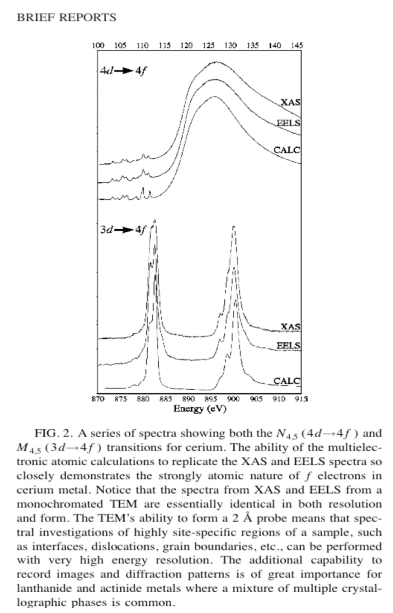

We've searched the literature and so far CANNOT find any U4f XAS. (We'll keep looking...) However, we have found U4f EELS and Th4f EELS, as shown above. From other experiments, we know that XAS and EELS can give the same answer, if the excitation energy is high enough in EELS. (See the example with Ce above.) The Th $4 f$ EELS shows that they are converging to the high energy limit above $1000 \mathrm{eV}$ and the $U$ $4 \mathrm{f}$ EELS was taken at $1200 \mathrm{eV}$, so it seems likely that this $\mathrm{U} 4 \mathrm{f}$ EELS should be the same as $U 4 f$ XAS. The U4f XAS TFY looks like it has the same peak structure as the $U 4 f \mathrm{fELS}$, if one discounts the region near $400 \mathrm{eV}$ with the Nitrogen contaminant. The fact that the $\mathrm{U} 4 \mathrm{f} X A S T F Y$ is so different than the U4f XAS TEY can be explained by stronger surface effects in TEY, which can also be seen in the O1s XAS, shown on the previous page. So, we think that this is pretty strong evidence that the U4f7/2 XAS TFY is legitimate. 


\section{Middle Energy Grating U 5d Region}

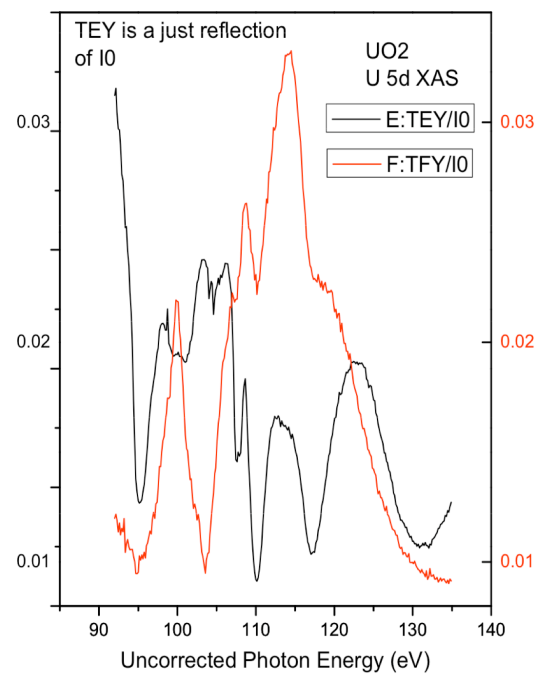

U 5d, van Kampen et al, PRA 61, 062706 (2000)

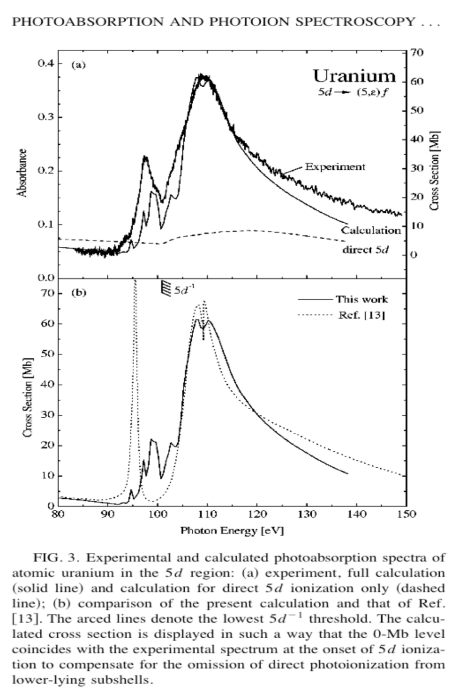

The above result is for an atomic $U$.

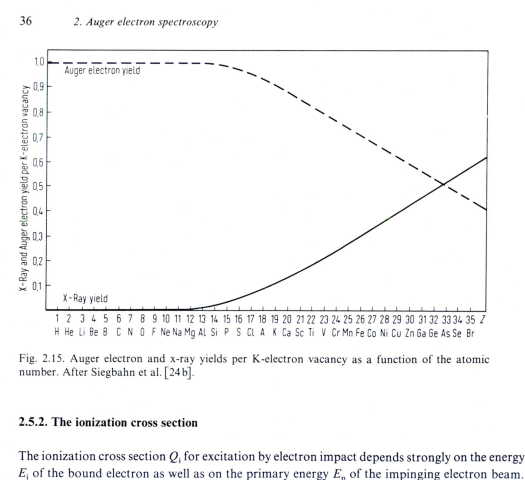

Ertl and Kueppers, "Low Energy Electrons and Surface Chemistry," Verlag Chemie, D-694 Weinheim, 1964, after K. Seigbahn et al, "ESCA Applied to Free Molecules," North Holland, Amsterdam, 1969

The issue of the weakness of the signal in TEY relative to TFY for the U4f and U5d may not be merely a sample size (signal vs background) or surface effect. (TEY is more surface sensitive.) It may also reflect the different fundamental efficiencies of TFY vs TEY. As shown in the figure above, TFY gains relative to Auger as $\mathrm{Z}$ increases, at least for the $\mathrm{K}$ edges shown. Of course, there can be edge specific effects: e.g., the U4d has a fairly strong TEY, the U4f and U5f don't. Nevertheless, Auger Emission drives TEY and X-ray emission is the source of TFY. The $\mathrm{z}$ dependence helps explain why the 01s has both TEY and TFY and the U seems to be losing the TEY but not the TFY.

Once again, we see that our TFY compares well with the literature and that the TEY has some sort of problem. Again, this indicates that the TFY is legitimate.

Conclusion: The TFY spectra are legitimate measures of the bulk electronic structure.

Tobin and Yu, LLNL 9 December $2010 \quad 14$ pages total Page 5 


\section{X-ray Absorption Spectroscopy of Uranium Dioxide}

Based upon LLNL-JRNL-454674

\section{Section 2. Monochromator Energy Calibration}

\section{High Energy Grating}

From the data on page 2 for the $\mathrm{U} 4 \mathrm{~d}$ region, it appears that the grating calibration is essentially dead on. This is not surprising, since the $3 \mathrm{~d}$ transition metal L edges are in this range and many people want to work at those edges. Thus, the ALS is fairly highly motivated to keep those calibrations up to date.

\section{Middle Energy Grating}

From the 01 s data on page 3 , for the edges seen with $1^{\text {st }}, 2^{\text {nd }}$ and $3^{\text {rd }}$ order light, it is clear that this grating needs to be calibrated. This is where the advantage of having three measurements of the same edge with first, second and third order light becomes so valuable.

Most grating errors, particularly for an SGM, which is just a high-energy SeyaNamioka, are caused by a misalignment of the grating zero, i.e. driven by a single $\Delta \lambda$. If this is true, then the errors should scale with E-squared.

$$
\begin{array}{ll}
E=h v=h c / \lambda & \text { with } v \lambda=c \\
d E=-h c(d \lambda) / \lambda^{2}=-E^{2}(d \lambda) / h c & \\
\Delta E=-E^{2}(\Delta \lambda) / h c \quad \text { with hc }=12400 \text { eV-Angstroms }
\end{array}
$$

$\begin{array}{llll}\text { Order } & \text { Uncorrected Energy(eV) } & \text { Unknown Value }(\mathrm{eV}) & \text { Energy Correction }(\mathrm{eV}) \\ & \mathrm{E} & & -\mathrm{E}^{2}(\Delta \lambda) / \mathrm{hc} \\ 1^{\text {st }} & 534.5 & \text { E0 } & -23.04 \Delta \lambda \\ & & & \\ 2^{\text {nd }} & 265.5 & (1 / 2) \mathrm{E} 0 & -5.68 \Delta \lambda \\ \mathrm{X} 2 & 531 & \text { E0 } & -11.36 \Delta \lambda \\ & & & \\ 3^{\text {rd }} & 176.5 & (1 / 3) \mathrm{E} 0 & -2.51 \Delta \lambda \\ \mathrm{X} 3 & 529.5 & \text { E0 } & -7.53 \Delta \lambda\end{array}$

Using the relations in red, there are three equations and two unknowns. We can back out the unknowns, E0 and $\Delta \lambda$, by a linear regression using $\mathrm{y}=\mathrm{E}$ and $\mathrm{x}=\mathrm{E}^{2} / \mathrm{hc}$. Then the slope of the line, $\mathrm{m}$, and the intercept, $\mathrm{b}$, give us the following.

$\mathrm{m}=-\Delta \lambda \quad$ and $\quad \mathrm{b}=\mathrm{E} 0$

From our analysis, E0 $=527 \mathrm{eV}$ and $\Delta \lambda=0.3$ angstroms, as shown on the following page.

Tobin and Yu, LLNL 9 December $2010 \quad 14$ pages total Page 6 


\section{X-ray Absorption Spectroscopy of Uranium Dioxide}

Based upon LLNL-JRNL-454674

\section{Middle Grating Calibration}

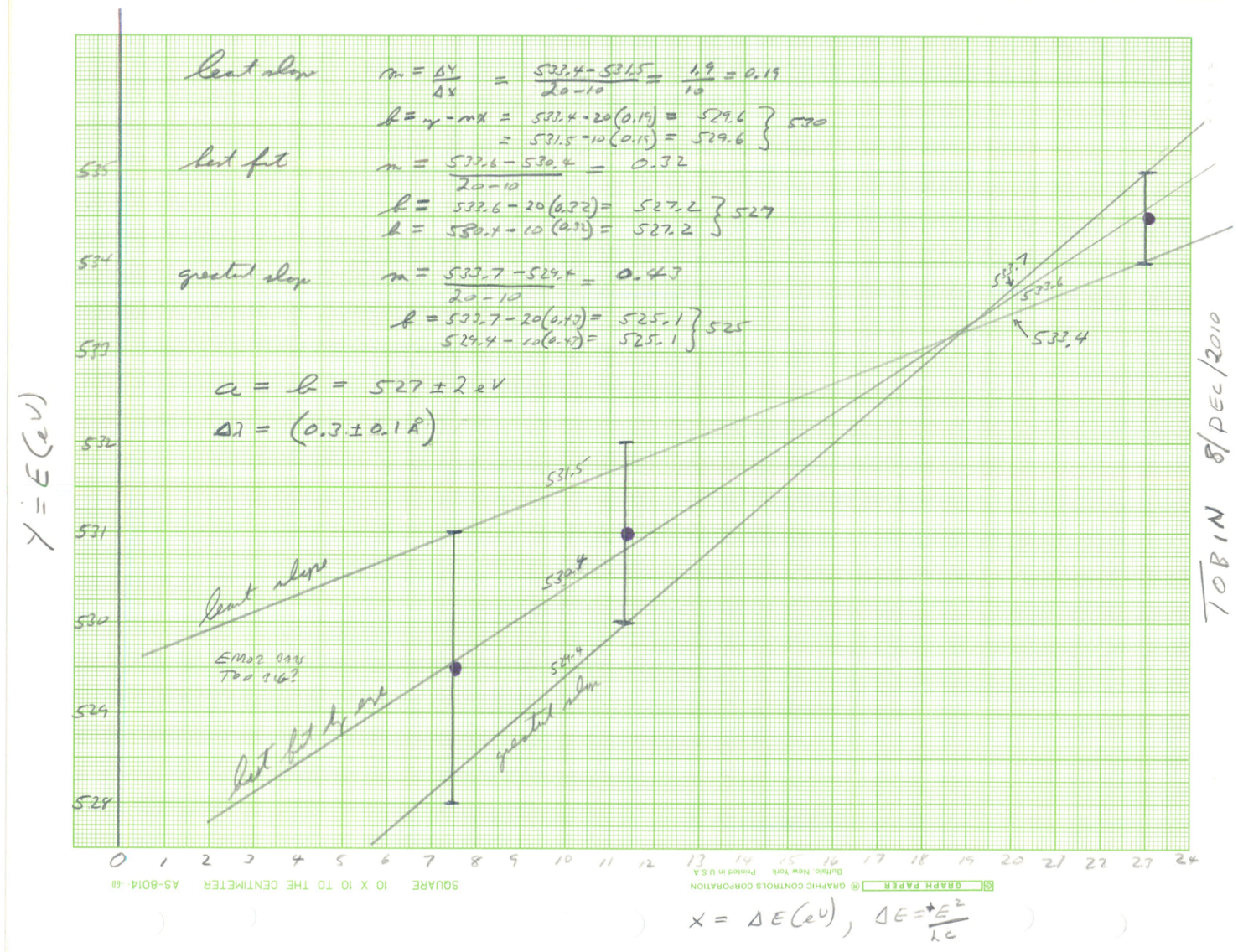

The error bars arise from the assumption of $+/-0.5 \mathrm{eV}$ error in the determination of the photon energies. This is probably too big. For the second and third order cases, because we double and triple the energies, respectively, the error also gets doubled or tripled. There should also be an error for $\mathrm{x}$, from propagating the energy error through the multiplication, but the y error is probably enough to give us a good significant figure estimate.

This is low tech, but it works.

When we shift the spectra, we get the result on the next page. 


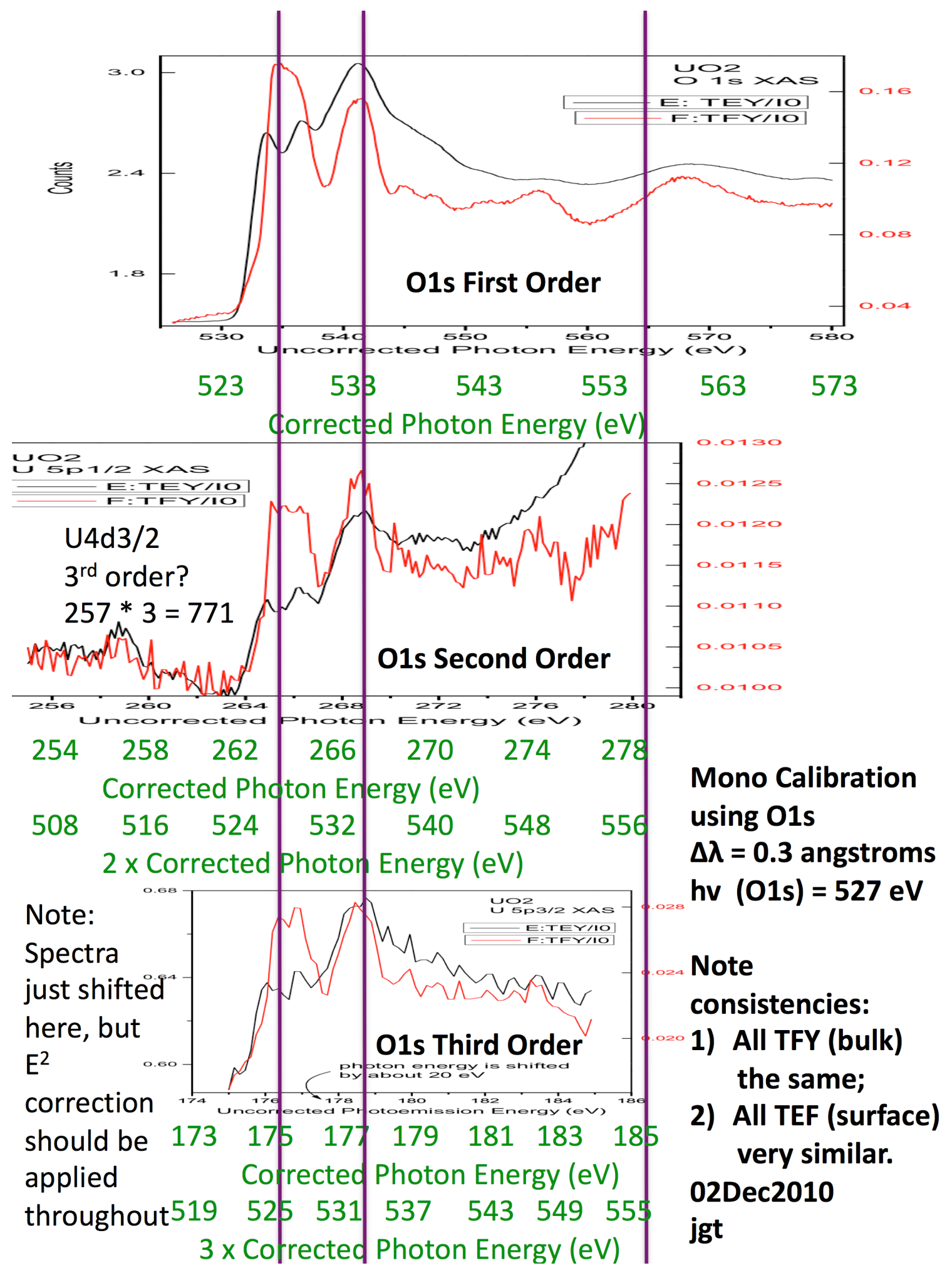

Of course, we can re-plot all of this to make it look nice, as shown on the next page. 


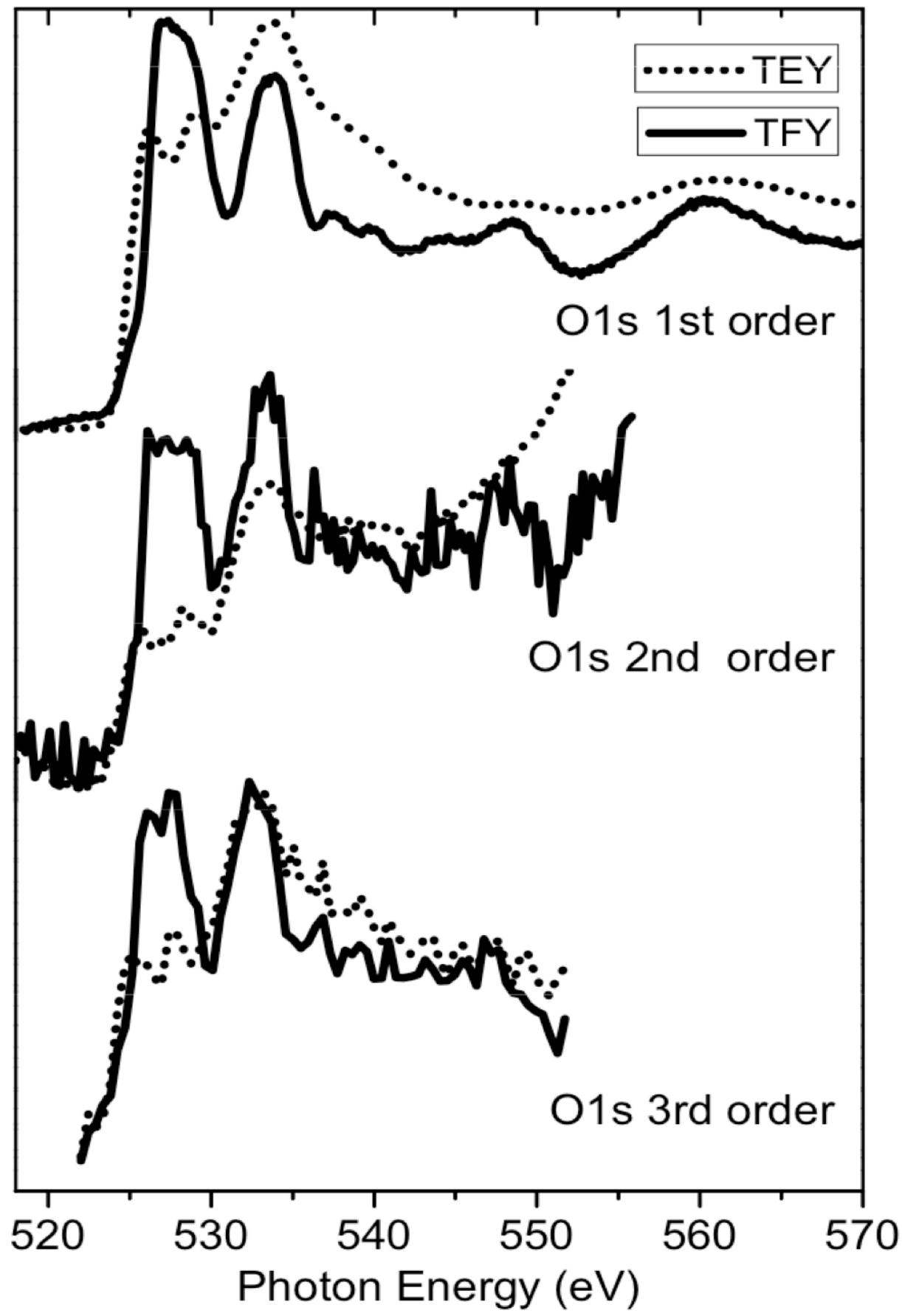

We find this quite convincing and hope that you do as well. 


\section{X-ray Absorption Spectroscopy of Uranium Dioxide}

Based upon LLNL-JRNL-454674

\section{Section 3. Comparison of Spectra}

Having a correct energy calibration for both the high and middle energy grating will then allow us to test the method that Sung Woo proposed in his talk. Note that while the U4d spectrum has the correct hv already, the U4f will need to shifted by the same relation as on page $\left.6, \Delta E=-E^{2}(\Delta \lambda) / h c=-(380)(0.3) / 12400\right)=-3.5$. Then, the binding energies for each core level are subtracted, using our XPS spectra for U02, which agree with the literature for UO2. Below are our spectra, with those from the literature. (All of these are in our MRS Proceedings, a copy of which is attached separately.)

Here are our XPS spectra, with the comparison to the literature values.
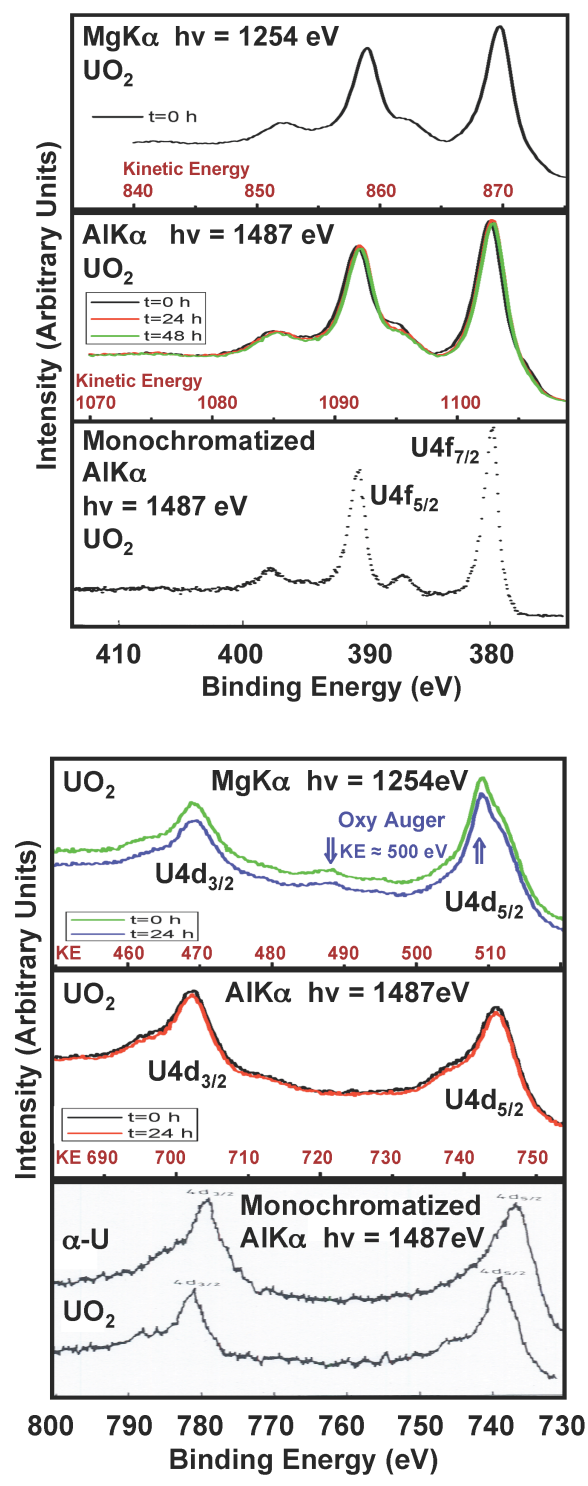

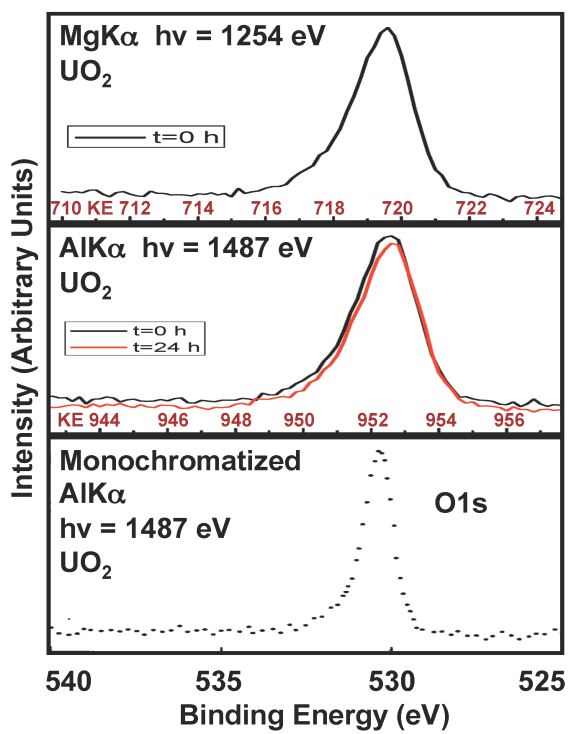

The article is JG Tobin, S-W Yu, BW Chung and GD Waddill, Mater. Res. Soc. Symp. Proc. 1264, 1264-Z11-06 (2010).

For U02, From Baer and Schones, Solid State Commun.

$\mathrm{BE}(01 \mathrm{~s})=530.5 \mathrm{eV}$ (from plot) $\mathrm{BE}(\mathrm{U} 4 \mathrm{~d} 7 / 2)=380 \mathrm{eV}$ (from plot)

For U02, From G.C. Allen et al, Phil. Mag. 1981

$\mathrm{BE}(\mathrm{U} 4 \mathrm{~d} 7 / 2)=380.25 \mathrm{eV}$ (average) $\mathrm{BE}(\mathrm{U} 4 \mathrm{~d} 5 / 2)=738.5 \mathrm{eV}$ (average)

The result of the comparison is shown on the next two pages. The second page is a blow-up of the first. 


\section{X-ray Absorption Spectroscopy of Uranium Dioxide}

Based upon LLNL-JRNL-454674

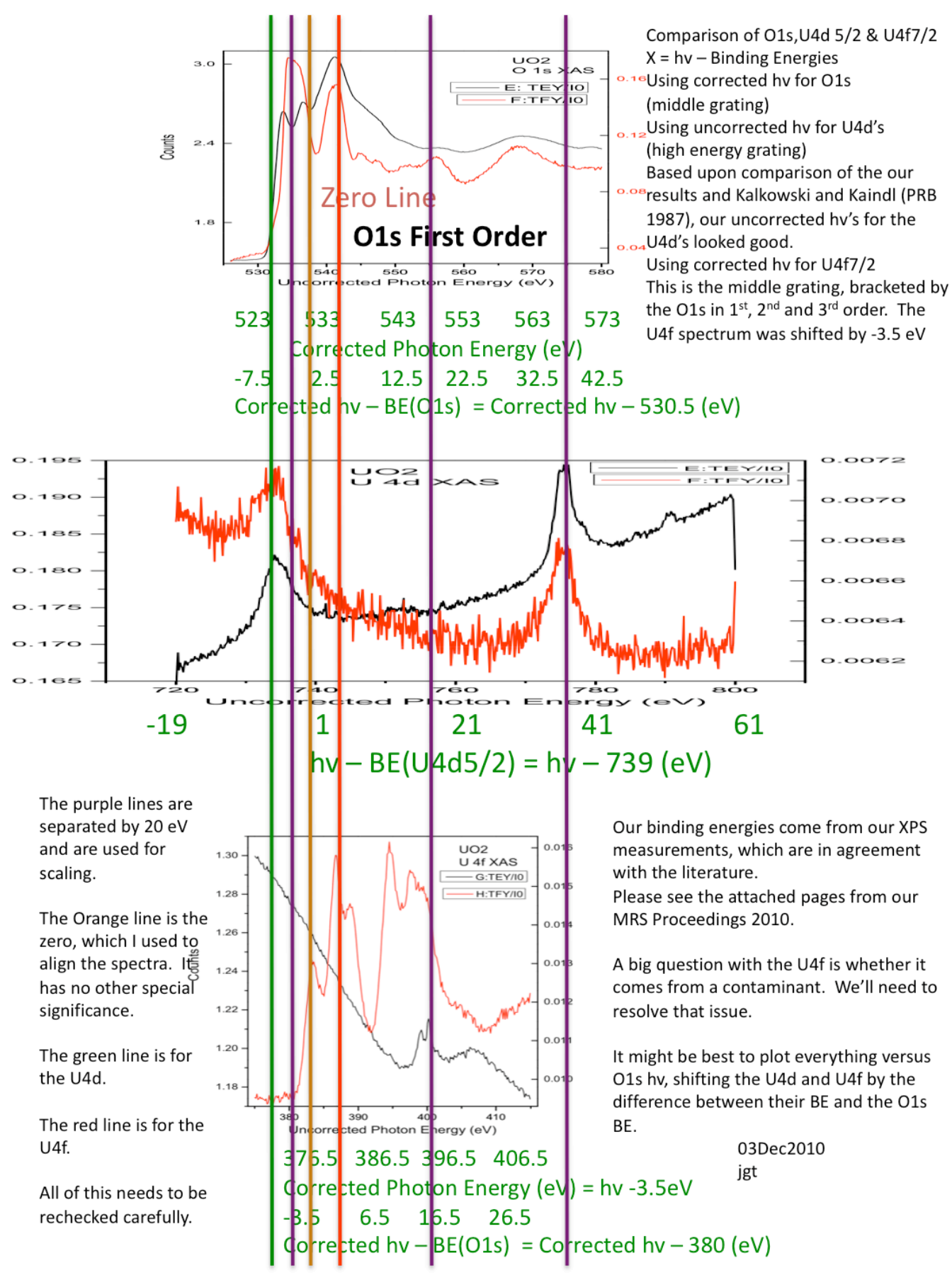

Tobin and Yu, LLNL 


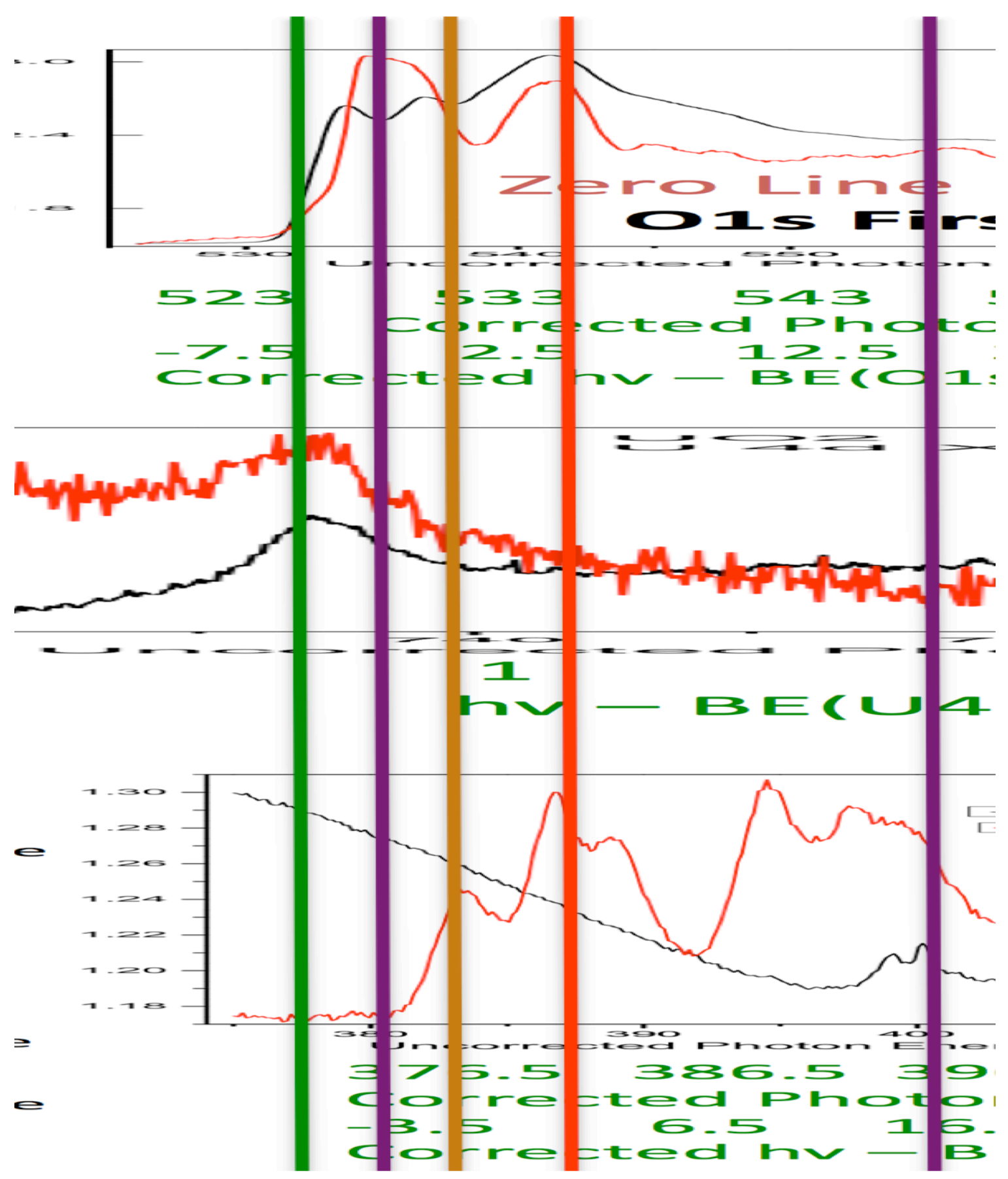

As you can see, we are back to something very close to what the figure on page 1 showed earlier. However, this is probably better. The U4d's have a huge lifetime broadening, giving rise to a Lorentzian, almost triangular, peak shape. The threshold may well be in the center of the white line peak, consistent with the alignment above. On the next page, we re-plot all of this. 


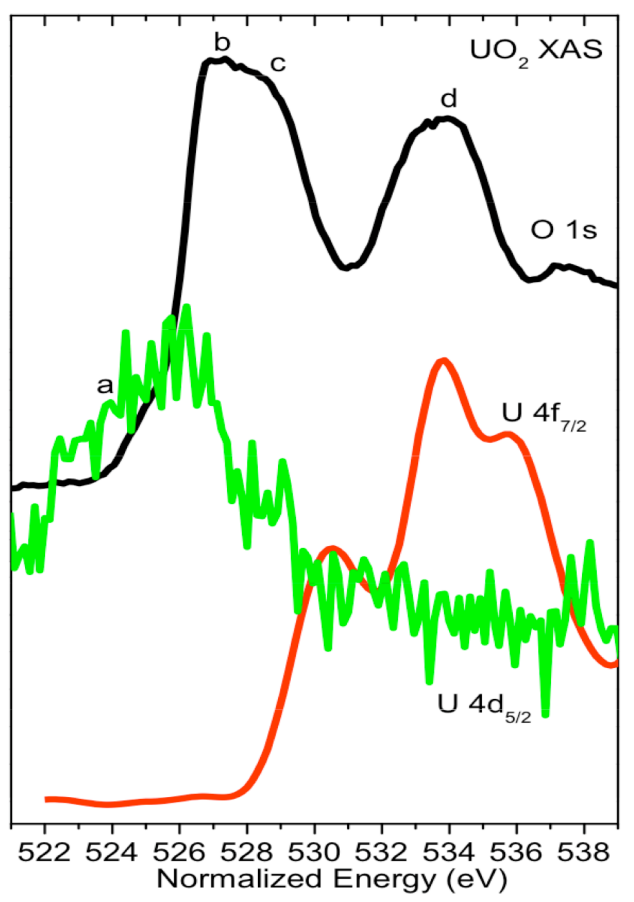

01s $\quad \mathrm{NE}=\mathrm{hv}(01 \mathrm{~s})$

U4f $\quad N E=h v(U 4 f)-[B E(U 4 f)-B E(01 s)]$

U4d $\quad \mathrm{NE}=\mathrm{hv}(\mathrm{U} 4 \mathrm{~d})-[\mathrm{BE}(\mathrm{U} 4 \mathrm{~d})-\mathrm{BE}(01 \mathrm{~s})]$
Here, we use "Normalized Energy," which is essentially the 01s photon energy.

Normalized Energy $=\mathrm{NE}$

$\mathrm{NE}=\mathrm{hv}-[\mathrm{BE}-\mathrm{BE}(01 \mathrm{~s})]$

with

hv $=$ Photon Energy

and

$\mathrm{BE}=$ Binding Energy from XPS

There was some concern voiced about subtracting binding energies (from XPS) from photon energies (from XAS), because of the different final states and concomitant effects such as screening, shielding, relaxation etc. (See below.)

XPS $=$ X-ray Photoelectron Spectroscopy

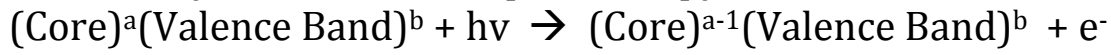

XAS = X-ray Absorption Spectroscopy

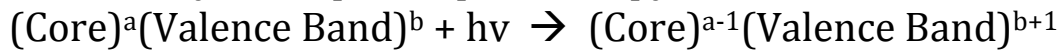

As the plot on the previous page suggests, all of these effects appear to cancel out. We can make that cancellation more obvious by using the Normalized Energy and subtracting a difference of XPS BE's, which will remove any question of reference points in the measurements. 


\section{X-ray Absorption Spectroscopy of Uranium Dioxide}

Based upon LLNL-JRNL-454674

\section{An Alternative Approach: Threshold Analysis}

While we are quite confident of the result derived from using photon energies and binding energies, there is a completely independent approach that will give almost exactly the same result. In the U4d spectra, there is a very strong lifetime broadening, which gives rise to a Lorentzian or triangular peak shape. The threshold is probably directly under the centroid of the peak. However, if one were to take a contrarian viewpoint, one could define the threshold as the beginning of the peaks spectral intensity. Then one could align all of the spectra based upon the thresholds, arguing that this represents the Conduction Band Minimum (CBM). If one follows through on this analysis, the result below is obtained, which is almost identical to the result on the previous page.

Either way, one ends up with something that looks very much like the XAS result on page 1.

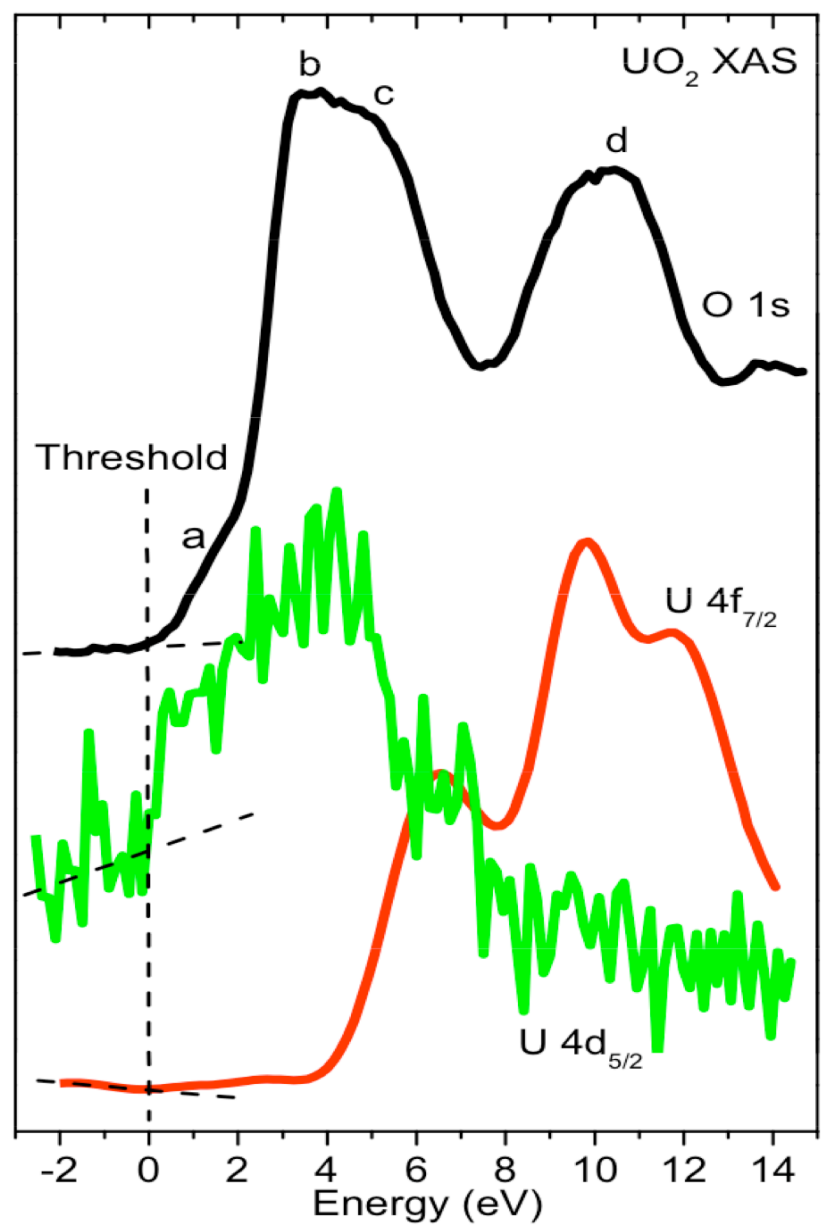

At this point, we see no show-stopping problems and propose that we continue with re-submission of the article, modifying it first to respond to the referee reports from PRL.

Tobin and Yu, LLNL 9 December $2010 \quad 14$ pages total Page 14 